\title{
Intracellular NOD2 activation promotes maturation and antigen-presenting functions of dendritic cells exposed to Porphyromonas gingivalis lipopolysaccharide.
}

\author{
Han Su${ }^{1}$, Xiang Yan², Wei Chen ${ }^{1}$, Ting Guo ${ }^{1}$, Zi-Tong Lin², Qin-Gang $\mathrm{Hu}^{2}{ }^{*}$ \\ ${ }^{1}$ Department of Stomatology, Jinling Hospital, Clinical School, Medical College, Nanjing University, Nanjing, PR China \\ ${ }^{2}$ Nanjing Stomatological Hospital, Medical School of Nanjing University, Nanjing, PR China
}

\section{Abstract}

\begin{abstract}
Objective: This study aimed to study the roles of Muramyl Dipeptide (MDP) which is an agonist of NOD2 in Dendritic Cells (DCs) exposed to Porphyromonas gingivalis lipopolysaccharide ( $\boldsymbol{P}$. gingivalisLPS) on maturation and antigen-presenting functions of DCs to provide experimentall evidences to explore the possible mechanism of DCs in periodontitis.

Methods: Flow cytometry was used to detect CD11c, MHC-II, CD80, CD86, and CD40 expression on DCs and ELISA was used to detect IL-12, IFN- $\gamma$, IL-10, and IL-13 secreted by DCs which were stimulated by MDP and $P$. gingivalis-LPS, respectively or in synergism. RT-PCR analysis was used to detect NOD2, TLR2, and TLR4 mRNA expression in DCs stimulated $0 y$ NDP and $P$. gingivalis-LPS, respectively or in synergism. CCK8 was used to assess CD4+ T cellsplviferation after co-cultured with DCs stimulated by MDP and P. gingivalis-LPS, respectively or sysergin and ELISA was used to detect IL-2, IFN- $\gamma, \Pi L-10$ and $\Pi \mathrm{L}-13$ secreted by these $\mathrm{T}$ cells.

Results: MDP had weak ability to stimulate DCs maturation but Mov could promote DCs maturation stimulated by $P$. gingivalis-LPS. MDP was NOD2 agonist to DO and $P$. gingivalis- LPS was TLR2 but not TLR4 agonist to DCs. MDP could facilitate TLR2 MRNA expression in DCs exposed to $P$. gingivalisLPS. The ability of MDP to promote DCs secreting cytkines was far below $\mathbb{P}$. gingivalis-LPS but MDP could promote the functions of Th2 cell-promatny DQs nduced by $P$. gingivalis-LPS. MDP could promote $\mathrm{CD}^{+} \mathrm{T}$ cells proliferation primed by $\mathrm{DCS}$ enest to $P$. gingivalis-LPS and ellevate the ability of DCs exposed to P. gingivalis-LPS to prime tho gells to Th2 cells.

Conclusion: Intracellular NOD2 in DCgacould be activated by MDP and this activation could promote maturation and the ability to prime Thocells tTh2 cells of DCs exposed to P. gingivalis-LPS.
\end{abstract}

\section{Introduction}

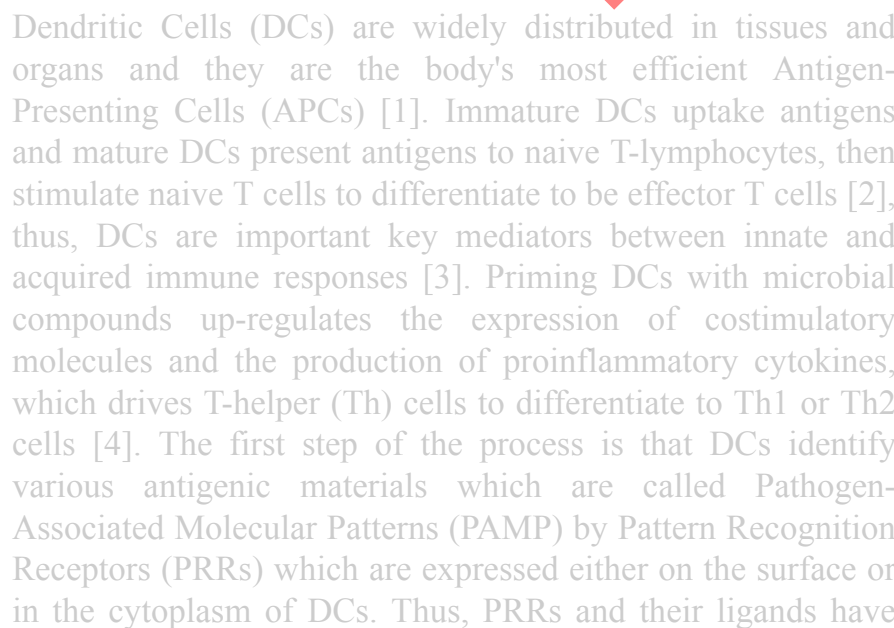

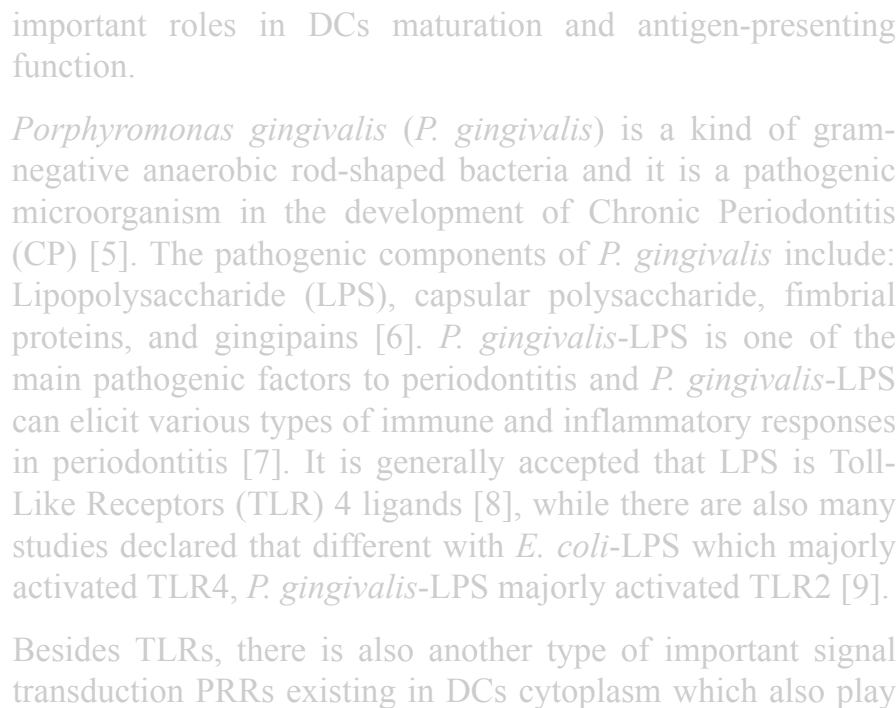

